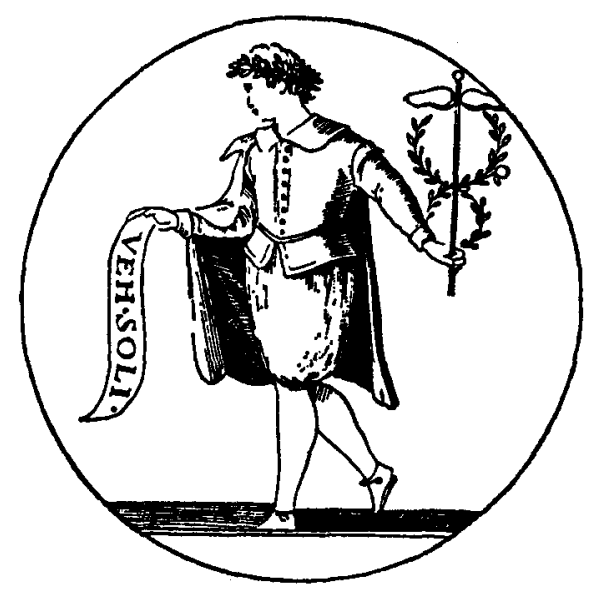

IMPRIMERIE FIRMIN-DIDOT

PARIS- MESNIL - IVRY

Le gerant : Pierre Junod 


\section{Annales Économies Sociétés Civilisations}

Revue bimestrielle fondée en 1929 par LUCIEN FEBVRE \&o $M A R C B L O C H$ Comité de direction : FERNAND BRAUDEL GEORGES FRIEDMANN - JACQUES LE GOFF EMMANUEL LE ROY LADURIE - CHARLES MORAZE

Secrétaire du Comité : PAUL LEUILLIOT Secrétaire de rédaction : MARC FERRO

$23^{\text {e }}$ ANNÉE No 6

NOVEMBRE - DÉCEMBRE 1968

\section{ETUDES}

I. WeLLMaNN

Zs. Kirilly et I. N. Kiss

E. L. Petersen

TRAVAUX EN COURS

G. Bors

S. Guilbert

\section{ENQUEATE OUVERTE}

Vie matérielle et comportement biologique (Bulletin $\mathrm{n}^{0}$ 17) :

G. Thuillier

\section{NOTE CRITIQUE}

A. TENENTI

Note sur les sources de l'histoire régionale de l'alimentation au $\mathrm{XIX}^{\mathrm{e}}$ siècle.

Esquisse d'une bistoire rurale de la Hongrie depuis la première moitié du XVIII siècle jusqu'au milieu du XIX'e siècle. Production de céréales et exploitations paysannes en Hongrie aux $X V I^{e}$ et $X V I I^{e}$ siecles.

La crise de la noblesse danoise entre I 580 et 1660.

Comptabilité et histoire des prix : le prix du“'froment à Rouen au XVe siècle.

A Châlons-sur-Marne au XVe siècle : un conseil municipal face aux épidémies.

NOTES BREVES ET PRISES DE POSITION

- Géographie et connaissance du monde

- Moyen âge ibérique, moyen âge européen

- Avocats et criminels

- Presse et information

- Sous-développement, sur-développement, croissance

COMPTES RENDUS

- Sur la " querelle des images"

- Finances et relations économiques à la fin du moyen âge

- Emigrants et immigrants

- L'Italie moderne et contemporaine

Rédaction : 20 rue de la Baume, PARIS-VIII' (BAL.45.45)

Administration : LIBRAIRIE A. COLIN, I03, bd St-Michel, PARIS-V

Comptes chèques postaux : Paris, $\mathrm{N}^{\circ} 21$ 335-25

Abonnements : France et Union française, $29 \mathrm{~F}$; Etranger, $35 \mathrm{~F}$

Le numéro de 208 pages : $6 \mathrm{~F}$ 


\section{INQUIRY \\ An Interdisciplinary Journal of Philosophy and the Social Sciences}

Edited by Arne Naess

VOLUME XI

Number 4

1968

Historical Objectivity and Value Neutrality

The Covering Law Model of Historical Explanation

Situational Logic and Covering Law Explanation in History

Norm and Law in the Theory of Action

Discussion :

Translation and Synonymy : Rosenberg on Quine

REview Discussion :

Arthur C. Danto, Analytical Philosophy of History, and Morton G. White, Foundations of Historical Knowledge

Fraser Cowley, A Critique of British Empiricism
James J. Leach

Stanley Paluch

Michael Martin

RUTH MaCKLIN

Philip L. Peterson

Leon Pompa

R. A. SHARpe

VOLUME XII

Number I

1969

Personal Identity in Spinoza

Language and Knowledge in Spinoza

Spinoza's Theory of Knowledge Applied to the Etbics

Freedom, Emotion, and Self-Subsistence: the Structure of a Small, Central Part of Spinoza's Etbics A Reconstruction of Basic Concepts in Spinoza's Social Psycbology

Spinoza on Power
Ruth L. SAW

G. H. R. PARKINSON

GuTTORM FLÖISTAD

Arne-Naess

JON WETLESEN

Robert J. MCShea

Published quarterly by Universitetsforlaget, P. O. Box 307 , Blindern, Oslo 3, Norway 


\section{Comparative Studies in Society and History}

Editors: SYLVIA L. THRUPP Professor of History, University of Michigan ERIC R. WOLF Professor of Anthropology, University of Michigan

Editorial Committee: ALBERT FEUERWERKER, MOSES FINLEY, CHARLES GIBSON, RAYMOND GREW, G. E. VON GRUNEBAUM, EDWARD SHILS, FREDERICK WYATT

Comparative Studies in Society and History is a forum for the presentation and discussion of research into problems of change and stability in human societies. It sets up a working alliance between specialists in all branches of the social sciences and humanities. Debate and review articles bring the general reader in touch with current findings and issues.

Ten volumes have already been published. Cambridge University Press will take over publication of Comparative Studies in Society and History from Volume II, 1969.

Articles on the following topics will appear in Volume II :

Ideology in Social Science, Ideology and Social Integration, Comparative Study of 'Feudal' Societies, Inheritance and Descent, Urbanism, Culture Contact, Charisma, Corruption, Healing Rituals.

Annual subscription $f_{3}$ net for four issues

Please write for a specimen copy

\section{CAMBRIDGE UNIVERSITY PRESS}

P.O. Box 92, London, N.W.I 


\section{AMERICA LATINA}

The regional social science journal in

Latina America

Sonve recent articles :

Gerrit Huizer : "Comunidades Agricolas" Internal Colinialism, and Agrarian Reform in Chile.

Jean Casimir : A Teoria dos polos de desenvolvimento e sua aplicagao ao caso dos paises subdesenvolvidos.

Maria Christina Salazar et alii : Aspectos de la deserción estudiantil en el Departamento de Sociologia de la Universidade Nacional de Colombia.

Eliseo Verón : Ideologia, y produción de conocimientos sociologicos en América Latina.

Hubert DE RONCERAx : Algunos aspectos de la ideología educacional en Haití.

SOCIAL SCIENCE NEWS

BOOK REVIEWS

ABSTRACTS

Published quarterly by the

LATIN AMERICAN CBNTER FOR RESEARCH IN THE SOCIAL SCIENCES

(under the auspices of UNESCO).

Subscription for four numbers : US $\$ 5.00$

Back numbers still available at : US \$ 2.00 each

The subscription includes, free of charge, the bimonthly bibliographical bulletin, Bibliografia, on the social sciences in Latin America.

Address : Caixa Postal 12 (ZC-02) Rio de Janeiro, Brazil. 


\section{T H E}

\section{Australian and New Zealand JOURNAL OF SOCIOLOGY}

The Sociological Association of Australia and New Zealand was founded in 1963 as a result of feeling among professional sociologists in Australia and New Zealand that the time had come for the formation of a national organization to advance the study of sociology in Australia. As part of its programme the Association will publish The Acstralian and New Zealand Journal of Sociology, twice a year in April and October beginning in 1965 . The journal will serve as an outlet for contributions by Australian and New Zealand scholars as well as articles by overseas sociologists writing about Australasian societies. A special feature of the journal will be the "News and Announcements section containing reports about the University departments where sociology is taught as well as news of sociological research in progress.

\section{PUBLISHED FOR THE SOCIOLOGICAL ASSOCIATION OF AUSTRALIA AND NEW ZEALAND \\ BY SIR ISAAC PITMAN AND SONS LIMITED, MELBOURNE}

The contents of the first two numbers will include the following papers : UNCERTAINTIES IN COMMUNITY STRATIFICATION

Athol A. Congalton

THE CHILD'S DISCOVERY OF SOCIAL GLASS, A. F. DAVIES RELIGION AND ASSIMILATION AMONGST DUTCH SETTLER IN WEST AUSTRALIA, J. E. Gough THE CORRELATION OF SOCIAL CLEAVAGES

AND THE INTENSITY OF GROUP CONFLICT, CharLes H. GRAY INTERRACIAL MARRIAGE IN AUCKLAND, John HARre A SOCIAL PROFILE OF CANBERRA, A.C.T., F. LANCASTER JoNes DURKHEIM'S SOCIOLOGY OF KNOWLEDGE, EUGENE KAMENKA POWER IN AUSTRALIA. R. S. PARKer SOCIOLOGY AND SOCIAL PHILOSOPHY, P. H. Partridge MAX WEBER'S ANALYSIS OF HERRSCHAFT, T. H. RIGBY A THEORETICAL BASIS FOR PROMOTING CHANGE

IN RURAL COMMUNITIES, JOAN TULLY REVIEW ARTICLE

ON THE MEÁSURE OF OCCUPATIONAL PRESTIGE, J. Allingham COMMENT, A. A. Gongalton

The annual subscriptions for both individuals and institutions, one volume of two numbers (each of sixty-four pages) will be : $£$ I. 10.0 (Australian), $£$ I. 5.0 (Sterling), 3.50 (U.S.A.) or the equivalent in the currency of the country to which the journal is addressed.

Subscriptions may be paid by cheques or Money Orders in the local currency and sent to the Honorary Business Manager who will arrange for their negotiation. Cheques, etc., should be made payable to the Sociological Association of Australia and New Zealand. The address is : MR. L. J. TIERNEY. Hon. Business Manager, Dept. of Social Studies, University of Melbourne, Parkville, N. 2, Victoria, Australia. 


\section{SOCIAL RESEARCH}

An international quarterly of political and social science published by the Graduate Faculty of the New School. The journal emphasizes interdisciplinary treatment of issues in the social sciences and seeks to maintain the humanistic tradition by integration with the approaches of philosophy and history. Special issues are focused. on problems which are examined by scholars in different fields of the social sciences.

\section{EDITORIAL AND BUSINESS OFFICE}

New School for Social Research

66 West Twelfth Street, New York 10011, N.Y.

Subscriptions : $\$ 10.00$ a year, domestic and foreign. Single copies $-\$ 2.50$

SOCIAL RESEARCH is published quarterly by the Graduate Faculty of Political and Social Science of the New School for Social Research, and appears in Spring, Summer, Autumn and Winter issues. All correspondence concerning manuscripts should be ddressed to Dr. Peter L. Berger. 


\section{UNITÉ NATIONALE ET MINORITÉS CULTURELLES}

Repenser la France :

La nouvelle littérature d'oc:

La question régionale : Jean-Marie DOMENACH et Robert LAFONT Jean B. SEGUY Pierre FOUGEYROLLAS, Robert LAFONT, Georges LAVAU, Vincent MONTEIL, Yves PERSON et Paul THIBAUD

Poèmes

Qu'est-ce qu'un mandarin?

Les anges de Kurbinovo
Jean FOLLAIN

HO HUU TUONG Robert MARTEAU

\section{JOURNAL A PLUSIEURS VOIX}

Les jeux olympiques. - La justice en Allemagne. - Le nouveau régime de la construction. - "CEdipe-Roi". - Les journées de musique contemporaine. Deux morts d'Espagne.

\section{GHRONIQUES}

Livres sur Mai J.-M. DOMENACH

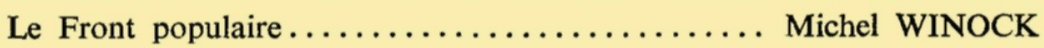

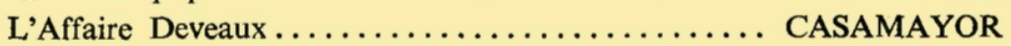

\section{DOCUMENT}

La "déposition" du Professeur Soulié

Ce numéro : France : ${ }_{7} \mathrm{~F}$.

Autres pays : $7,40 \mathrm{~F}$.

\begin{tabular}{|c|c|c|c|c|}
\hline \multirow[t]{2}{*}{ Abonnements } & \multicolumn{2}{|c|}{ FRANCE } & \multicolumn{2}{|c|}{ AUTRES PAYS } \\
\hline & 6 mois & I an & 6 mois & $I$ an \\
\hline Ordinaire...$\ldots \ldots \ldots \ldots \ldots \ldots$ & $F_{30}$ & F 58 & $\mathrm{~F}_{33}$ & 63 \\
\hline Soutien $\ldots \ldots \ldots \ldots \ldots \ldots \ldots \ldots$ & $\mathrm{F}_{40}$ & F 80 & $\mathrm{~F}_{40}$ & F 80 \\
\hline Luxe numéroté............ & F 55 & F IIO & F ss & F r ro \\
\hline
\end{tabular}

ESPRIT - i9, rue Jacob - Paris-6e.

C.C.P. Paris $1154-51$. 


\title{
QUADERNI DI SOCIOLOGIA
}

\section{Trimestrali}

\author{
Comitato direttivo : \\ Nicola Abbagnano, Franco Ferrarotti, Luciano Gallino, Angelo Pagani, \\ Alessandro Pizzorno, Pietro Rossi, Tullio Tentori, Renato Treves. \\ Direttore responsabile : Franco Ferrarotti. \\ Redattore : Luciano Gallino.
}

VOLUME XVII - $x_{968}$ - N. I-2 - NUOVA SERIE

EDITORIALE

A. Anfossr, Principi impliciti nella teoria del Scientific Management

F. Remottr, La concezione dei sistemi in Lévi-Strauss

L. Benzi - M. MARchetrr, Bibliografia classificata di Sociologia della letteratura

E. Moriondo, Una ricerca di antropologia giuridica in Arabia Saudita

G. BRAvo, Incontro con sociologi ungheresi

R. Treves, Socialismo e Marxismo

\section{SCHEDE}

PANORAMA DELLE RIVISTE

NOTIZIARIO DELL'ASSOCIAZIONE ITALIANA DI SCIENZE SOCIALI

Redazione e Amministrazione : Casa editrice Taylor, via Valeggio 26, ToRINo.

Telefono 584.15s - CC postale 2/34389.

Abbonamenti : Italia L. 4000 , estero L. 6000.

un fascicolo arretrato L. 2000 . Un fascicolo separato L. I 200. 


\title{
MINER VA
}

A Review of Science, Learning and Policy

Editor : Edward Shils

\begin{tabular}{lll}
\hline VOLUME VI & Winter 1968 & NUMBER 2 \\
\hline
\end{tabular}

\author{
ARTICLES \\ The Situation of the Universities in Greece \\ GEORGE HANIOTIS \\ An Example of Demograpbic Accounting: The Scbool Ages \\ Richard and Grovanna Stone and Jane Gunton \\ Universities in Turkey \\ Osman OKYar
}

\section{REPORTS AND DOCUMENTS}

Hands off the Universities?

ERIC AsHBY

The Medium of Instruction in Indian Higher Education

\section{CORRESPONDENCE}

CHRONICLE

MINERVA, Ilford House, 133-135, Oxford Street, London, W. I.

Please send me the current issue MINERVA AES/22

I wish to subscribe to MINERVA (delete as necessary)

Single copy 7s. 6d. - \$1.25. Annual subscription 30s. - \$5.

Name

Address

MINERVA, Ilford House, 133-135 Oxford Street, London, W. r. 


\section{DIOGEेNE}

Revue internationale des sciences humaines

Rédacteur en chef : Roger CaILlois

\section{$\mathrm{N}^{\circ} 64$}

\section{Octobre - décembre I 968}

\section{NOUVELLE ACTUALITÉ DU MARXISME}

Theodor Adorno
Herbert Marcuse
Jean Hyppolite
Éric J. Hobsbawm
Maxime Rodrnson
Anouar ABdel-malek
Abdallah LARour

Robert C.TuCKER

\author{
Marx est-il démodé? \\ Réexamen du concept de révolution \\ Le "scientifique et l'idéologique" dans une perspective \\ marxiste \\ L'apport de Marx à l'bistoriographie \\ Sorijlogie marxiste et idéologie marxiste \\ Marxisme et sociologie des civilisations \\ L'intellectuel du Tiers monde et Marx, \\ ou encore une fois le probleme du retard bistorique \\ Marx et la fin de l'bistoire
}

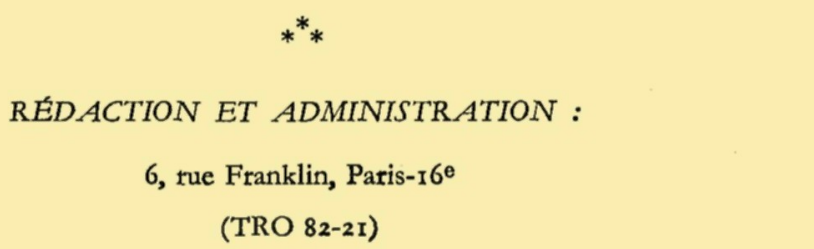

Revue trimestrielle paraissant en trois langues : anglais, espagnol et frangais.

L'édition française et publiée par la Librairie Gallimard, s, rue Sébastien-Bottin, Paris- $7^{\mathrm{e}}$.

Les abonnements sont souscrits auprès de cette maison (C.C.P. 169-33, Paris).

Prix de vente au numéro : $5,50 \mathrm{~F}$.

Tarif d'abonnement : France, $20 \mathrm{~F}$; Étranger, 25,50 F. 


\section{SOCIAL SCIENCE \\ Information}

\section{sur les sciences sociales}

An international journal in English and French published six times yearly by the International Social Science Council.

$\begin{array}{lll}\text { VOLUME VHI NUMBER I } & \text { FEBRUARY I968 }\end{array}$

\section{FROM THE CONTENTS}

ARTICLES BY

Carl Göran Andrab

STEIN ROKKAN

Pierre Bourdieu

Pablo Gonzalez Casanova

Seymour MARTIN LipSET
Popular movements in Sweden : report on a mass-data research project.

Centre formation, nation-building and cultural diversity : report on a symposium organized by Unesco.

Intellectual field and creative project.

Etablissement d'un plan de developpement en sciences sociales.

The possible political effects of student activism.

AND THE SECTIONS : Theory and Metbods, New Metbods in History, Organization of Researcb and Training, Inventories and Bibliographies.

Subscription price $\$ 18$ or 80 F yearly payable to the publisher : Mouton \& Co. 39 avenue Philippe-Auguste, 75-Paris Ire.

Special rates for students and individual members of national disciplinary associations : \$12 (r $60 \mathrm{~F}$.

Editorial Address : International Social Science Council,

6, tue Franklin, 75-Paris-16e. 


\section{REVUE FRANÇAISE}

$\mathrm{DE}$

\section{SOCIOLOGIE}

publiée par le

\section{CENTRE D'ÉTUDES SOCIOLOGIQUES \\ CENTRE NATIONAL DE LA RECHERCHE SCIENTIFIQUE}

\section{Vol. IX, No 1. Janvier-Mars I968}

HaNS ZeIsel

KLAUS Liepelt

Vladimir N. ChOUbKine

GuY Michelat

et Jean-Pierre Thomas

Haroun Jamous
L'école viennoise des rechercbes de motivati...

Esquisse d'une typologie des électeurs allemands et autricbiens Le choix d'une profession. Résultats d'une enquête sociologique auprès de jeunes de la région de Novosibirsk

Contribution à l'étude du recrutement des écoles d'Officiers de la Marine (1945-1960)

Éléments pour une théorie sociologique des décisions politiques

Vol. IX, No 2. Avril-Juin 1968

TRAVAUX DE L'INSTITUT DE SOCIOLOGIE URBAINE

Rédaction

CENTRE D'ÉTUDES SOCIOLOGIQUES

82 , rue Cardinet,

PARIS-XVIIO

\section{Abonnements}

Éditions du C.N.R.S.

I 5, quai Anatole-France, PARIS-VIIe

4 numéros trimestriels et I numéro spécial

L'abonnement :

France : $40 \mathrm{~F}$ Étranger : 8 \$

Le numéro spécial double : is $\mathrm{F}$

Éditions du Centre National de la Recherche Scientifique - C.C.P. PARIS 906I-II. 


\section{PAST AND PRESENT}

First published in 1952

No 4I (Dec. 1968)

includes the following articles :

Peter Garnsey : Legal Privilege in the Roman Empire.

K. LeYSER : The German Aristocracy from the Ninth to the early Twelfth Century. A Historical and Cultural Sketch.

C.S.L. Davies : The Pilgrimage of Grace Reconsidered.

C.H. GeORGe : Puritanism as History and Historiography.

JoHN R. Gillis : Aristocracy and Bureaucracy in Nineteenth Century Prussia.

E. Abrahamian : The Crond in Iranian Politics x905-1953.

As from January 1969, Past and Present will appear quarterly.

Subscription rates will be :

Annual Subscription 40 s. (\$6)

Single issue $20 \mathrm{~s} .(\$ 3)$

Reduced Annual Subscription for Students 20 s. $(\$ 3)$

All past issues available - Classified Table of Contents free on request.

Write : Business Manager, PAST AND PRESENT, Corpus Christi College, Oxford. 


\section{Les lettres nouvelles}

\section{NOVEMBRE-DÉCEMBRE I968}

\section{TEXTES :}

Zdenek LORENC

George Perec

Léonie BRUEL

James STERN

Arnaldo Calveyra

Pierre Peuchmaurd

Viviane Forrester

Boris NICOLAEVSKI
Appels (traduit du tchèque)

La consultation

Dialogue

Rencontre avec Lowry

Il m'arrive encore de mourir

Le poète ordinaire

La moindre transparence

L'assassinat de Kirow

\section{ETUDES :}

Théâtre d'avant-garde aux U.S.A., Raymond Aron, Stanislaus Joyce, Virginia Woolf, un sonnet retrouvé de Nerval, etc.

à paraître en FÉVRIER 1969 :

Numéro spécial sur la JeUne Littératture GreCQue

Les bureaux de la rédaction sont transférés aux ÉDITIONS du MERCURE 26, rue de Condé, Paris-VI

MED, 86.77

Même adresse pour les abonnements

Abonnement simple à la revue : $30 \mathrm{~F}$ (France et étranger)

Abonnement complet ouvrages et revue : $80 \mathrm{~F}$ (France et étranger) 


\title{
REVISTA ESPAÑOLA DE LA OPINION PUBLICA \\ (Trimestral)
}

\author{
Director \\ Salustiano DEL CAMPO URBANO \\ Subdirector Ejecutivo \\ Luis GONZALEZ SEARA \\ Consejo de redacción :
}

Alfonso Alvarez Villar

Juan Beneyto Ferez

José Castillo Castillo

José Cazorla Perez

Juan Diez Nicolas

Gabriel Elorriaga Fernandez

Alberto Gutierrez Reñon
José Jimenez Blanco

Juan J. Linz de Gracia

Carmelo Lison Tulosana

Enrique Martin Lopez

Amando de Miguel Rodriguez

Francisco Murillo Ferrol

José R. Torregrosa Peris

Jorge Xifra Heras.

\section{Secretario : \\ José Sanchez Cano \\ Secretario Adjunto : \\ Matia Teresa Sancho Mendizabal \\ Sumario $\mathrm{N}^{\circ}{ }_{14}$ (oct.-diciembre 1968)}

ESTUDIOS :

La sociedad caótica : consecuencias de la revolución morfológicosocial

Las vacaciones del español y el problema del tiempo libre

El lugar del cine en el proceso de comunicación del mañana

La intogración social de la prensa y las instituciones de autacontrol

Caracteristicas de los tele-espectadores japoneses de espacios dramáticos

Ensayo de tipificación de los sin opinión

ENCUESTAS :

Philit H. Hauser

Luis Gonzales Seara

Alphons SilbermanN

Bernard VoyenNe

José María Véra, S. J.

José Luis Martin Martinez

INFORMACION :

\section{Cuestiones de actualidad}

Luis Gasca :Bibliografia mundial del " comicu

a) Cuestiones religiosas

b) Politica internacional

c) Politica interior

d) Sociología social

e) Tiempo libre

Bibliografia

CONGRESOS Y REUNIONES

\section{Suscripciones}

ESPANAA :

Número suelto $\ldots \ldots \ldots \ldots \ldots \ldots \ldots \ldots \ldots \ldots \ldots \ldots \ldots \ldots \ldots \ldots \ldots \ldots \ldots \ldots \ldots, 90,00$ Ptas.

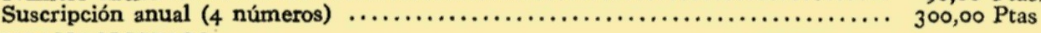

HISPANOAMERICA :

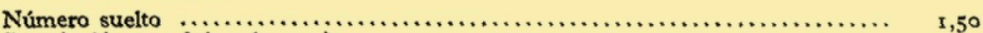

Suscripción anual (4 números) $\ldots \ldots \ldots \ldots \ldots \ldots \ldots \ldots \ldots \ldots \ldots \ldots \ldots \ldots, \quad 5,50$

OTROS PAISES

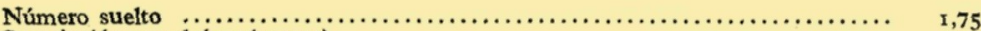

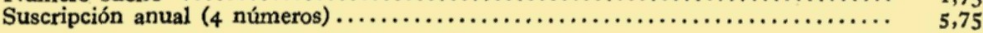

REDACCION Y ADMINISTRACION

Paseo de la Castellana, 40 - MADRID - Teléfono 2-76-87-16 


\title{
Zeitschrift für allgemeine Wissenschaftstheorie
}

\section{Journal for General Philosophy of Science}

\author{
Herausgegeber von \\ Alwin Diemer . Lutz Geldsetzer . Gert König \\ In Zusammenarbeit mit \\ Abteilung für Wissenschaftstheorie, Philosophisches Institut \\ der Universität Düsseldorf
}

Die Zeitschrift erscheint halbjäbrlich mit je 160 Seiten. Jabresabonnement $D M, 6,-$ Einzelbeft DM 32, -

Mit der ZeItsChrift Fur ALLgemeine Wissenschaftstheorie wird ein philosophisches Unternehmen gestartet, das über die bisherigen wissenschaftstheoretischen Arbeiten hinausgreift. Es stellt einen Brückenschlag zwischen Natur-, Sozial- und Geisteswissenschaften dar und zielt damit auf eine „Philosophie der Wissenschaft(en)“. Die Herausgeber haben sich entschlossen, für den deutschen Titel den traditionell gewordenen Begriff „Wissenschaftstheorie“ $z u$ verwenden.

Die ZeItsChrift FÜR ALLGEMEINE WISSENSChaftSTHEORIE enthält :

\section{Aufsätze}

Berichte zur allgemeinen wissenschaftstheoretischen Diskussion der verschiedenen Sprachgebiete

\section{Rezensionen}

Einen bibliographischen Teil :

neueste Literatur und Spezialbibliographien

Sie wendet sich nicht nur an Wissenschaftstheoretiker und Philosophen, sondern auch an alle Fachvertreter in den Natur-, Sozial- und Geisteswissenschaften, denen es darum geht, ihren Standort im Rahmen angrenzender und übergreifender Fachgebiete zu bestimmen und von diesen Erkenntnisse und methodische Anregunged zu gewinnen.

\section{$Z u$ beqieben durch Ibre Bucbbandlung}

FRANZ STEINER VERLAG GMBH · WIESBADEN 


\title{
TABLE QUINQUENNALE
}

\author{
des tomes I à $\mathrm{V}$ \\ $1960-1964$
}

Al B ERT, Hans.

Zur Logik der Sozialwissenschaften, V 2.

A NDERSON, Bo and ZRLDITCH, Morris Jr. Rank Equilibration and Politics, V $\mathbf{~}$.

A N D R E S K I, Stanislav.

Conservatism and Radicalism of the Military, II r.

Aron, Raymond.

Science et conscience de la société, I r.

Les sociologues et les institutions representatives, I r.

Sociologie allemande sans idéologie? I x.

Classe sociale, classe politique, classe dirigeante, I 2.

Les etapes de la croissance économique, $I_{2}$.

La mitraillette, le char d'assaut et l'idée, II I.

La définition libérale de la liberté, II 2 et $\mathrm{V}_{2}$.

Quelques problèmes des universités françaises, III $\mathbf{x}$.

Macbl, Power, Puissance : prose démocratique ou poésie démoniaque, $\mathrm{V}$ r.

BA HRDT, Hans Paul.

Zur Frage des Menschenbildes in der Soziologie, II x.

BAILBY, Frederick G.

Traditional Society and Representation : a case study

in Orissa, I $\mathbf{~}$.

Closed Social Stratification in India, IV I.

BARBU, Zevedei.

"Chosisme ", IV 1.

BEN-DAvID, Joseph and ZLOCzOW BR, Awraham.

The Idea of the University and the Academic Market Place, II 2.

Universities and Academic Systems in Modern Societies, III 1.

BENDIX, Reinhard.

Social Stratification and the Political Community, I 2. Public Authority in a Developing Political Community : the case of India, IV .

Bétgille, André. A note on the Referents of Caste, V .

ВовттснвR, Erik.

Soziale Marktwirtschaft oder Wohlfahrtsstaat? Die deutsche Erfahrung, II 2.

BoTTOMORE, T. B.

The ideas of the Founding Fathers, I 1 .

American Heretics, I 2.

Recent Theories of Development, III 2.

BOUDON, Raymond.

Quelques fonctions de la formalisation en sociologie, IV 2.

BrIGgs, Asa.

The Welfare State in Historical Perspective, II 2.

B R OTz, Howard.

Functionalism and Dynamic Analysis, II I.
Crozinr, Michel.

Classes sans conscience ou préfiguration de la société sans classes, I 2.

De la bureaucratie comme système d'organisation, II I

Pouvoir et organisation, $\mathrm{V}$ I.

DA HRBNDORF, Ralf.

Demokratic und Sozialstruktur in Deutschland, I I.

Aspekte der Ungleichheit in der Gesellschaft, I 2.

Amerikanische Orthodoxie, I 2.

Uber einige Probleme der soziologischen Theorie der

Revolution, II r.

European Sociology and the Americain Self-Image,

II 2.

Starte und Offenheit der deutschen Universität, III 2. Amba und Amerikaner: Bemerkungen zur These der Universalität von Herrschaft, $\mathrm{V}$ x.

Dorg, R. P.

Japan as a Model of Economic Development, V I.

DrgitzBL, Hans Peter.

Selbstbild und Gesellschaftsbild. Wissenssoziologische therlegungen zum Image-Begriff, III 2.

BtzronI, Amitai.

International Prestige, Competition and Peaceful Coexistence, III $\mathrm{t}$.

FALLERS, Lloyd.

Political Anthropology in Africa, IV 2.

Fieischa anN, Eugène.

Métamorphoses wébériennes, $\mathbf{V} \mathbf{~}$.

De Weber à Nietzsche, $V_{2}$.

FrBUND, Julien.

Le concept de public et l'opinion, $V_{2}$.

GRLL N R, Emest.

Patterns of Rural Rebellion in Morocco : tribes as minorities, III 2.

Graman 1, Gino and Sil v zRt, Kalman.

Politics, Social Structure and Military Intervention in Latin America, II $\mathrm{r}$.

GroHs, Gerhard.

Zum Gesellschaftsbild revolutionärer und nachrevolutionärer Architektur in Frankreich, IV 2.

Wandlungen der Sozialen Rolle des Kunstkritikers, $\mathrm{V} 2$.

HALSBX, A. H.

British Universities, III $\mathbf{x}$

HoFPMANN, Stanley.

Observations sur la crise politique française, I 2.

How A RD, Michael.

Civil-Military Relations in Europe and the United States : a bibliographical note, II $\mathbf{r}$.

L A N C A S TBR, Lorraine.

Crédit, épargne et investissement dans une économie

« non monétaire ", III $x$. 
LEF ÈVRE-PONTALIS, J.-B.

Refiexions sur le langage de la psychanalyse, IV 2.

LEF OR T, Claude.

Machiavel jugé par la tradition classique, I I.

Lгснтнатм, George.

Class and Hierarchy : a critique of Marx? V 1 .

Lroszr, Seymour Martin.

Party Syotems and the Representation of Social Groups, I 1.

LocxwoOD, David.

The \& New Working Class ", I 2.

LocxMANN, Thomas and BERGBR, Peter. Social Mobility and Personal Identity, V 2.

Marshalr, T. H.

The Welfare State : a sociological interpretation, II 2.

MAт в R, Adrian C.

Some Political Implications of Community Development in India, IV $x$.

MEnTon, Robert $\mathrm{K}$.

Resistance to the Systematic Study of Multiple Discoveries in Science, IV 2.

Nв в в L, William $\mathbf{H}$.

Roles as a Criterion for a General Theory of Caste, V I.

Ozconomo, Constantin.

La version américaine du métier des armes, II I.

PIRKER, Theo.

Von der Herrschaft über Menschen zur Verwaltung der Dinge, $\mathbf{V}$.

Przzorno, Alessandro.

Lecture actuelle de Durkheim, IV $\mathbf{x}$.

Poprtz, Heinrich.

Soziale Normen, II 2.

Pr $\mathbf{x}$, Lucian W.

Armies in the Process of Political Modernization, II r.
R E T A A U D, Jean-Daniel.

La steurité sociale en France : du conflit doctrinal a l'affrontement des intétes, II 2.

Économie gtntraliste? III 2 .

Ro\&zRTS, Bryan.

Politics before Leisure : David Riesman's changing place in American sociology, IV $x$.

Rox $\times$ AN, Stein.

Mass Suffrage, Secret Voting and Political Participation, II $\mathbf{~}$.

Ross, Amold.

On Individualism and Social Responsibility, II $x$.

Social Mobility and Social Values, V 2.

Rosst, Paolo.

Les arts mecaniques et la science nourelle, IV 2.

Ross I, Pietro.

Contributions italiennes $\$$ la théric sociologique, III 1.

RUNCIMAN, W. G.

Problems of Research on Relative Deprivation, II 2. Charismatic Legitimacy and One-Party Rule in Ghana, IV $\mathrm{r}$.

R U w $\mathbf{z}$, Nicolas.

La linguistique générale sujourd'hui, V 2.

Sскмі т, Hannelore.

Die deutsche Exekutive 1949-1960, IV 1.

Sigarst, Christian.

Bine Brwiderung auf einen Aufatz von Ralf Dahrendorf, V 2.

TAlMON, Yonina.

Pursuit of the Millenninm : the relation between religious and social change, III $\mathbf{x}$.

TOURAIN E, Alain.

Travail et organisations, III I.

Pour une sociologie actionnaliste, $\mathrm{V}$ I.

Taow, Martin.

The Democratization of Higher Education in America, III 2.

I I Industrial Society and Representative Government. 2 A la recherche des classes perdues.

II I Le sabre et la loi. $\sim_{2}$ On the Welfare State.

III I-2 Universităt im Umbau : Anpassung oder Widerstand?

IV I In Quest of Political Participation. 22 Trois études sur la science.

V I Organisation der. Macht, Macht der Organisation. $a_{2}$ Tocqueville, Marx, Weber.

VI I Simulation in Sociology. $\sim_{2}$ Armed Forces and Society in Western Europe.

VII I Aliénation et stucture or conscience and consciousness. $\infty 2$ On Suicide.

VIII I Paradoxes of Transitional Societies. $\sim_{2}$ Sympathy for Alien Concepts.

IX I Weber et Durkheim : le solitaire et le chef d'école. $\sigma_{2}$ Zur Problematik der Modernisierung or on the concept of modernity. 